\title{
DIFTERIA MALIGNA
}

\section{Drs. MAFALDA RIZZARDINI y OSCAR UNDURRAGA \\ Cátedra de Pediatria del Prof. Julio Menegbello. \\ Hospital "Manuel Artiarán". Santiago.}

El presente trabajo tiene por objeto dat a conocer algunas caracteristicas clínicas de la forma más severa de difteria, a través del análisis de 19 casos fallecidos en el Servicio de Infecciosos del Hospital "Manuel Arriaran" desde 1946 a 1954.

Del total de enfermedades infecciosas hospitalizadas en el Servicio, durante este lapso de tiempo, un $9,1 \%$ corresponde a difterias y de ellas, alrededor del $5 \%$, a difterias malignas (Gráfico $N^{0} 1$ ). Queremos hacer notar la incidencia del cuadro a que nos referimos. Relacionándola con las cifras dadas por autores nacionales ${ }^{1}$ nuestra incidencia es mayor y se acerca, por tanto, a las obtenidas por autores extranjeros 2.3 .4 . Estas formas de difteria junto con los cuadros laringeos obstructivos, que fueron motivo de una publicación anterior ", y las complicaciones cardiacas, constituyen graves problemas de tratamiento y son las causas más frecuentes de muerte por esta enfermedad.

Casi todos los Tratados de Pediatría y de Enfermedades Infecciosas se han pre- ocupado de la forma maligna de la difteria, describiendo una serie de tipos y subtipos.

En general se acepta que existen tres síndromes malignos en la difteria: el precoz o de Verdoux, el secundario o de Marfan y el tardío o de Grenet y Mezard. Nos referiremos al primer cuadro, llamado también difteria hipertóxica, ya que los otros dos síndromes corresponden a complicaciones más o menos tardias de la difteria, que también matan, frecuentemen. te al enfermo, pero no ya por toxemia sino que, en el caso del sindrome de Marfan, por compromiso cardiovascular con insuficiencia cardíaca y en el de Grenet y Mezard por parálisis invasoras.

La difteria maligna es una enfermedad bien característica y absolutamente diferente de la difteria común, lo que hacia decir a Marfan "que podrían mirarse como enfermedades distintas.

Todos los enfermos de difteria maligna, dice Leunda ', se parecen; podrán diferir uno de otro por diferentes grados de

\section{GRAFICO ME 1}

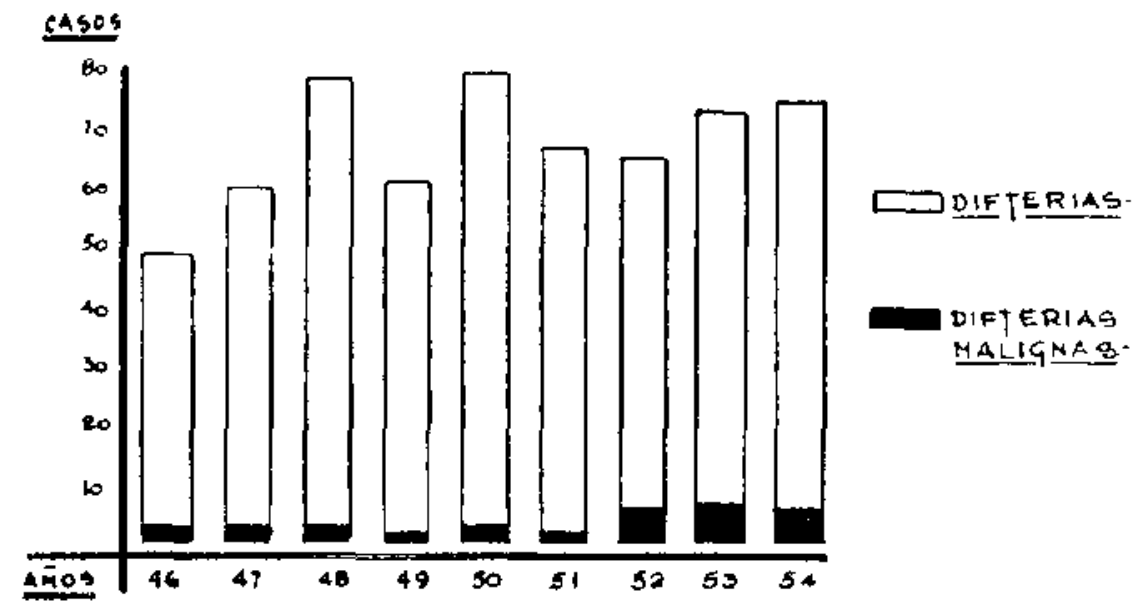

\footnotetext{
FRECUEMCIA DE DIFTERIAS HOSPITA. LIZADAS EN EL SERVICIO DE INFECCIOSOS DEL HOSPITAL ARRIARAN.
} 
lesiones locales, de vecindad o generales, pero hay hechos comunes que permiten reconocerla.

Podriamos definir como maligna la difteria hipertóxica que lleva a la muerte en los primeros días de enfermedad. Aunque este cuadro tóxico no se presente en el primero o segundo día, algunos elementos clínicos permiten pronosticar la aparición, en breve plazo, del síndrome de toxemia que ensombrece el pronóstico y lleva frecuentemente a la muerte.

Es por eso que con este autor, de reconocida experiencia, creemos que no es práctica la descripción de una serie interminable de tipos de difterias malignas, reservando esta denominación para el cuadro gravísimo que pasamos a analizar.

\section{MATERIAL}

En la tabla siguiente, hemos consignado algunas características generales. La edad de nuestros pacientes fluctuó entre 3 y 12 años, siendo la mayoría mayores de 6 años. En cuanto al sexo no hubo diferencias importantes: 9 enfermos correspondian al sexo masculino y 10 al femenino. Se presentó la enfermedad en cualquier época del año, pero fué más frecuente en primavera, otono e invierno, lo que coincide con la prevalencia estacional de la difteria en 'general ${ }^{8}$.

Entre el comienzo de la enfermedad y el ingreso al Servicio, transcurrieron 2 días como mínimo, con un promedio de 3,4 días. Los síntomas más destacados por su intensidad, obtenidos mediante la anamnesis, fueron: aumento de volumen cervical, que llamó la atención en 8 casos; el decaimiento fué un signo evidente en $4 \mathrm{y}$ el sindrome purpúrico y los vómitos en 2 respectivamente. No consignamos aquí otros sintomas o signos que habiéndose presentado frecuentemente en el periodo de prehospitalización, los consideramos comunes a cualquier otro tipo de difteria.
La sintomatología general y local, al ingreso, se esquematiza en el Gráfico N" 2. En él podemos apreciar que todos nuestros pacientes presentaron al ingreso, evidente compromiso del estado general, siendo muy intenso en 12 . El psiquis es. tuvo comprometido en 10 pacientes, caracterizándose este compromiso por somnolencia, embotamiento y hasta profundo sopor. Algunas veces se observó períodos de sopor alternados con periodos de excitación psicomotora (3 casos). Este compromiso general y psíquico se fué intensifícando en los días que precedieron a la muerte.

En general nuestros enfermos evolucionaron con temperatura baja y la curva térmica no presentó caracteristicas especiales. La mayoría de las veces se observó moderada elevación térmica el $1^{\circ} \mathrm{y}$ el $2^{\circ}$ dia, en los siguientes, hubo temperatura normal $n$ hipotermia.

En el examen segmentario destacaremos los hechos más llamativos por su frecuencia e intensidad.

La palidez de la piel es un signo casi constante en la difteria " pero en nuestro material adquirió particular intensi. dad en 17 casos, siendo de menor importancia aunque siempre notable, en el resto. La deshidratación, que se describe con frecuencia en las toxemias graves, estuvo presente en 6 de nuestros casos sin que este signo llamara la atención por su intensidad. La tendencia a las hemorragias se describe clásicamente como característica de este tipo de difteria ${ }^{10}$. En 6 de nuestros niños hubo algún tipo de púrpura: petequial en 3, esquimótico en 1 y estuvieron presentes los dos tipos simultáneamente en 2. No fué raro encontrar equímosis y puntos sangrantes en el sitio de las inyecciones, característica ésta que se observó la mayoría de las veces, al final de la evolución. Con menos frecuencia vimos vómitos de tipo hemorrágico (2 casos), en cambio, la epistaxis se presentó en casi todos los enfermos, ya sea an-

TABT.A

CARACTERISTICAS GENERALES EN 19 NIROS CON DIFTERIA MALIGNA

\begin{tabular}{|c|c|c|c|c|c|c|c|c|c|}
\hline \multicolumn{2}{|c|}{ Edad (atios) } & \multicolumn{2}{|c|}{ Sexo } & \multicolumn{2}{|c|}{$\begin{array}{l}\text { Tiempo de evo ución } \\
\text { prehospita aria (dias) }\end{array}$} & Sintom & destacador & \multicolumn{2}{|c|}{ en la onampesis } \\
\hline-6 & $60+$ & $\mathbf{M}$. & F. & $-d e 4$ & tat & $\begin{array}{l}\text { Aumento de } \\
\text { vo:nmen } \\
\text { cervical }\end{array}$ & $\begin{array}{l}\text { Deczi- } \\
\text { miento }\end{array}$ & Vómitos & $\begin{array}{l}\text { Sindrome } \\
\text { purpúrico }\end{array}$ \\
\hline 4 & 15 & 9 & 30 & 11 & $\mathbf{8}$ & 8 & 4 & 7.2 & 2 \\
\hline
\end{tabular}


GRAFICO NO2

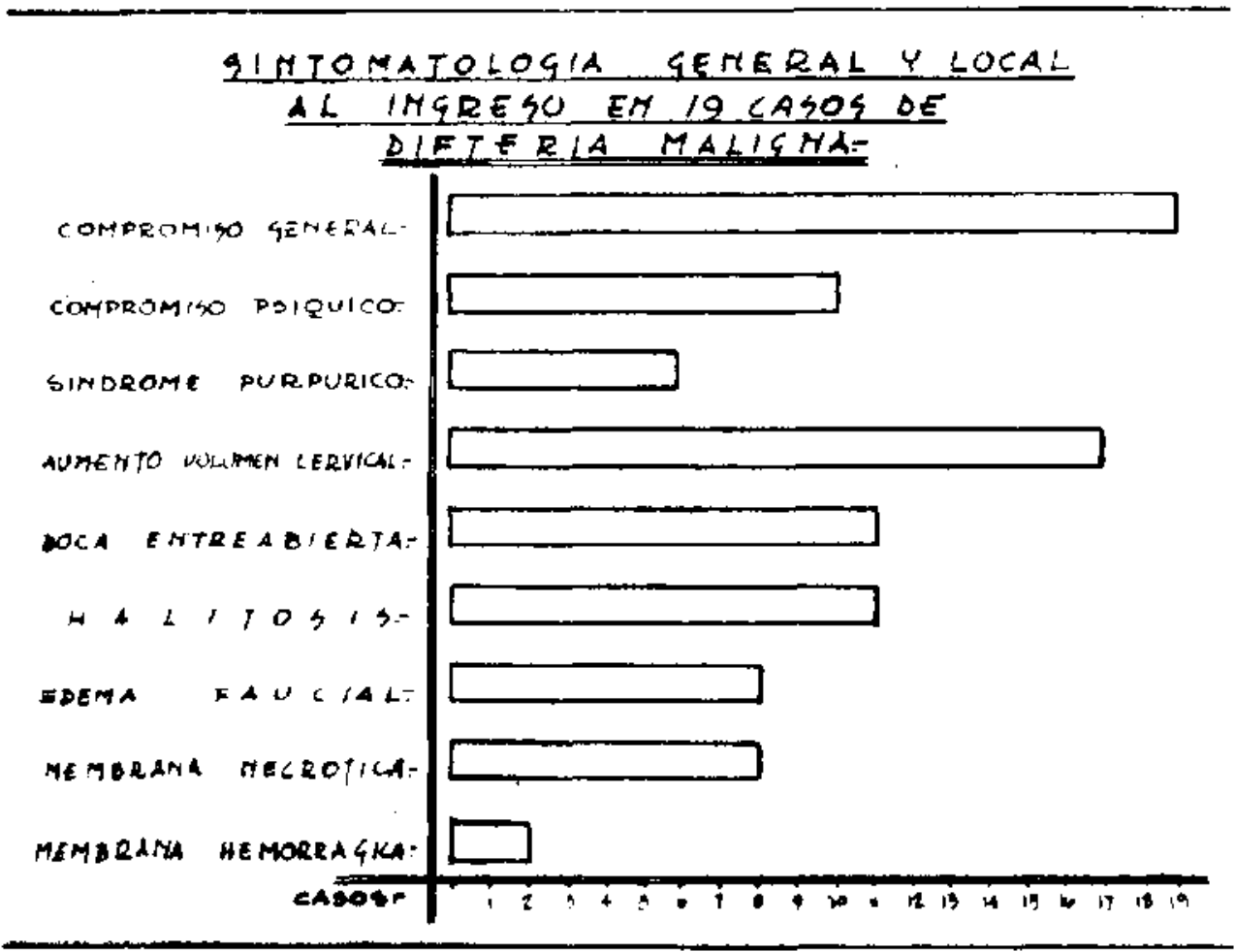

tes o durante la hospitalización. Fué copiosa y persistente en 2 casos.

Il de nuestros pacientes se mantenian con la boca entreabierta, emitiendo un olor nauseabundo; en el resto la halito. sis no fué tan intensa, aunque siempre estuvo presente. En el examen faringeo destacó el edema faucial que se consignó como importante sólo en B, aunque creemos que es un signo casi constante en las difterias graves y malignas. Aspecto necrótico o hemorrágico de la pseudomembrana se observó en 10 niños; en el resto, el aspecto no fué diferente al de cualquier otro tipo de difteria, pero siempre el exudado fué muy extenso.

Aumento de volumen cervical de cierta magnitud se observó en 17 niños, llegando a adquirir las características de cuello proconsular en 12. Rara vez fué posible delimitar bien los ganglios infartados; generalmente el edema periganglionar impidió hacerlo, palpándose sólo una masa pastosa la mayoría de las veces poco dolorosa.

Las manifestaciones cardíacas de la difteria serán objeto de una próxima publicación, de modo que nos limitaremos a ex- poner algunas características del examen cradiovascular en nuestro material. Al ingreso, la taquicardia fué la regla, siendo ésta superior a 140 por minuto en 9 casos. No siempre la taquicardia fué un signo que se mantuviera durante toda la evolución, llamando la atención en 5 enfermos, la aparición brusca de bradicardia, uno o dos días antes de la muerte. El apagamiento de los tonos estuvo presente en 12 oportunidades y fué un signo importante por su intensidad. Tonos arrítmicos se anotó en 3 casos (una arritmia completa y dos arritmias extrasistólicas). La gravedad de los pacientes impidió practicar exámenes que complementaran los hallazgos clínicos. Sólo fué posible practicar ECG. en 4 niños y de éstos, uno resultó normal, otro reveló un bloqueo de rama derecha y en 2 hubo bloqueo de rama izquierda. En los 3 electrocardiogramas alterados se informó: signos evidentes de daño miocárdico.

La presión arterial al ingreso fué normal en 6 casos; en 3 , hubo hipotensión y no se tomó la presión en el resto. Sólo en 1 niño apreciamos signos evidentes de shock al ingreso, pero durante Ia evolu- 
CRAFICO NO 3

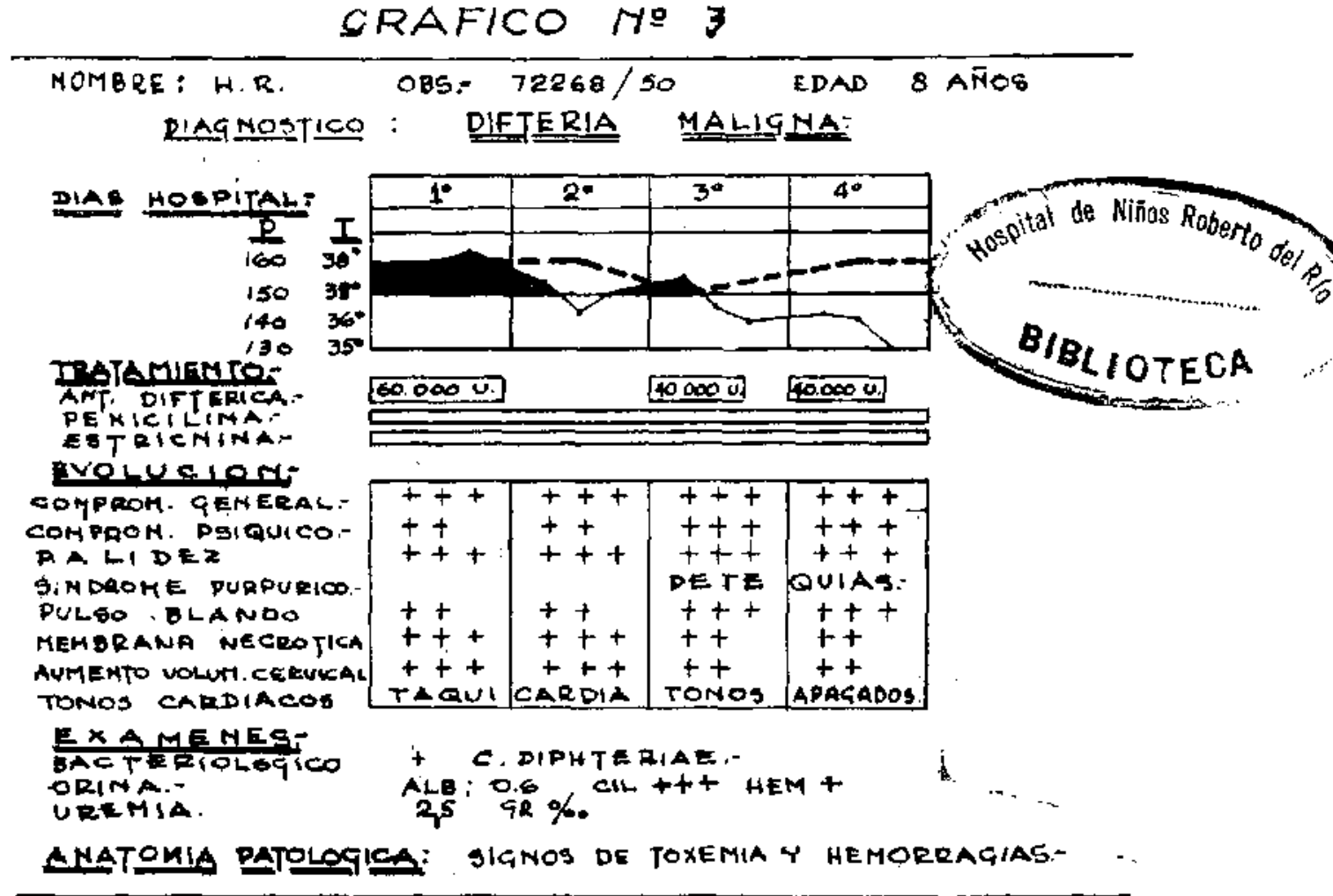

ción, en 17 niños, se abservó la aparición de este síndrome como precursor del deceso.

Es un hecho frecuente encontrar un sindrome urinario en cualquier tipo de difteria. En 9 de nuestros pacientes se practicó un examen de orína, encontrándose desde indicios de albúmina hasta un máximo de $3 \mathrm{gr}$. por mil. La cilindruria fué también una alteración casi constante, comprobándose en 6 exámenes. La hematuria se presentó en 8 enfermos y siempre fué microscópica. A este síndrome se agregó, en 4 oportunidades, franca oliguria, llegando a la anuria.

En 6 pacientes se dosificó la urea en la sangre, encontrándose en 5 cifras superiores a $0,50 \mathrm{gr} . \%$. La cantidad máxima fué de $2,50 \mathrm{gr}$. $/$ c. Este examen se practicó la mayoria de las veces el dia del ingreso.

La sedimentación globular estuvo moderadamente elevada en nuestro grupo y el hemograma no tuvo nada de característico. En 6 casos en que se practicó este último examen, la leucocitosis fué la regla, siendo el número de leucocitos superior a 15.000 , en 4. La desviación a la izquierda fué intensa en toda la serie, observándose siempre marcadas alteraciones toxodegenerativas en los neutrófilos. Las plaquetas eran normales en 5 enfermos, observándose en el otro, franca disminución de ellas $(38.000)$. Sólo en 1 niño se investigó tiempo de coagulación y sangría, siendo ambos normales, a pesar que clínicamente se evidenció un intenso sindrome hemorragíparo.

En el gráfico No 3 hemos resumido un caso que desde el punto de vista clínico, consideramos típico de la enfermedad que nos ocupa.

H. R. Observación NP 72.268/50. Niña de 8 años que ingresa al Servicio el 3 de Junio de 1950 por una enfermedad que se habtia iniciado 2 dias antes con disfagia, fjebte, vómitos, epistaxis y atumento progresivo de volumen del cuello. En el examen físico se comprueba: temperatura de $38^{\circ}$; pulso $160^{\circ}$. muy blando. regular; presión 99/60. Parece muy grave, por momentos excitada $y$ llama la atención la gran palidez. de los tegumentos. Permanece con la boca entreabierta, despidiendo un olor repugnante. La faringe está muy edematosa, cubierta de extensas pseudomembranas de aspecto necrótico. Hay gran tamefacción del cuello. Los tonos cardiacos. aunque tápidos. son de tonalidad $y$ timbre normales. E! exament de orina practicado al segundo dia, da albaminuria $0,60 \mathrm{gr} . \% \mathrm{ke}$ intensa cilindruria $y$ disereta hematuria. El cultivo dc secreción faringea foé positivo para ol C. diphteriae. 
Se colocó $60.000 \mathrm{U}$. de Antitoxina diftérisa el primer dia; al día siguiente se inyectó $40.000 \mathrm{U}$. $y$, dos dias más tarde. $40.000 \mathrm{U}$.

Se administró $300.000 \mathrm{U}$. de Penicilina Procaína cada 12 horas, con un total de 2.700 .000 U. Adamás se indicó estricnina $0.5 \mathrm{mgr}$. cada 6 horas, extracto suprarrenat, percorter, luminal, cafeina, suero glucosado en fleboclisis y se insistió en el reposo absoluto. Todas estas medidas no lograron modificar el curso de la enfermedad, observándose agravamiento de los signos de toxemia en los dias sucesivos. Al segundo dia de hospitalización aparecen signos francos de colapso y más tarde. algunas petequias en el cuelfo y parta alta del tórax. Fallece al $4^{\circ}$ dia de estada $\left(6^{\circ}\right.$ de enfermedad).

El informe anatomopatológico es el siguiente: Difteria tatingea. Hemorragia perequial de la pirl. Numerosas hemorragias subpleurales $y$ del pericardio visceral. Tumefacción turbía de los túbulos secretores renales. Es decir, se encontraton lesiones de toxemia y hemorragias propias de este tipo de enfermedad.

Todos nuestros enfermos recibieron antitoxina diftérica, la mayoría de las veces, en dosis única y por via intramuscular. Hubo 6 casos en que se usó, tanto la vía endovenosa como la intramuscular $y$ al mismo número de pacientes se inyectó más de una dosis. No observamos síntomas imputables a la administración de la antitoxina por vía endovenosa, pero tampoco hubo diferencias en cuanto al resultado final, cuando se usó de preferencia esta vía. Las dosis empleadas en nuestro grupo fluctuaron entre 40 y 160 mil unidades, con un promedio de $80 \mathrm{mil}$.

Usamos antibióticos en la difteria para eliminar los gérmenes de asociación, especialmente el estreptococo hemolítico y para disminuír la producción de toxina, actuando directamente sobre el $\mathrm{C}$. diphteriae, germen muy sensible a la mayoria de estas drogas ${ }^{1.12}$. Se usó penicilina prácticamente en todos los casos, sola o asociada a estreptomicina. Dos niños recibieron eritromicina, con el fin de adquirir experiencia clínica con este antibiótico tan activo "in vitro", contra el B. de Loeffler ${ }^{13}$. Las pautas terapéuticas en cuanto a antibióticos se refiere, fueron variadas, no observándose diferencias cua'quiera que fuere el esquema utilizado. Se administró cortisona a dos enfermos, pensando actuar sobre la toxemia de la difteria con igual éxito que el obtenido por Smadel 14 en la tifoidea. El $30 \%$ de nuestros niños recibió estricnina desde el primer día, en dosis que no sobrepasarọn los 2 mgr. diarios. En algunos casos se administraron vitaminas $\mathrm{C}$ y $\mathrm{K}$ y se adoptaron las medidas de enfermería adecuadas, como ser el reposo absoluto, asi como aquéllas usuales tendientes a combatir el colapso, la excitación, la obstrucción respiratoria y las alteraciones del equilibrio hidrosalino. Ante un cuadro tan grave, cualquier ensayo terapéutico encontró justificación, a pesar de ello, en nuestro material el pronóstico fué siempre fatal.

Se hizo autopsia en 17 de nuestros 19 pacientes y en todos se confirmó el diagnóstico de difteria. En el 50\% las membranas no estaban sólo localizadas en la cavidad bucofaringea, sino que se exten. dían hacia la laringe ( 15 casos), tráquea ( 1 caso) e incluso hasta las ramificaciones bronquiales ( 3 casos), lo que confirmó la apreciación clínica en cuanto a extensión de las membranas se refiere. Signos evidentes de miocarditis se observaron en 5 casos, revelados en el examen microscópico en mayor o menor grado de infiltración histiocitaria del intersticio $y$ en una oportunidad por necrosis de la fibra miocárdica. En 4 niños que clínicamente presentaron evidentes signos de compromiso cardíaco, dos de ellos con ECG. francamente anormal, la autopsia no reveló a\}teración miocárdica alguna. Es nosible que en estos casos la duración de la presunta miocarditis fuese lo suficientemente breve como para no hacerse visible el examen microscópico.

En el riñón se encontró en forma frecuente ( 9 casos), degeneración epitelial de los túbulos colectores y secretores.

La degeneración de la capa fascicular de la corteza suprarrenal, descrita por Rich (citada por ${ }^{3}$ ), no fué comprobada en nuestro material. Sólo en una autopsia se encontró hiperhemia capilar difusa en esta glándula.

\section{COMENTARIO}

El cuadro nosológico de la difteria maligna constituye para nosotros una entidad con características propias, que permite reconocerla desde los primeros días de su evolución, asignándole, por lo tanto, un pronóstico diferente al de cualquier otra forma de difteria.

Para formular el diagnóstico de difteria malígna esperamos encontrar la mayoria de los siguientes elementos carac- 
terísticos de la enfermedad a que nos referimos:

1. Signos evidentes de toxemia que se manifiestan especialmente por el compromiso del estado general y, sobre todo, psiquico.

2. Pseudomembranas invasoras de as. pecto necrótico o hemorrágico.

3. Síndrome bemorragíparo.

4. Aumento acentuado de volumen del cuello.

5. Compromiso renal y cardiovaseular.

En nuestra experiencia, la mortalidad fué de $100 \%$. Esta cifra coincide con la experiencia de autores franceses ${ }^{16}$, pero se aleja bastante de la de otros, como Bazán ${ }^{2}$ quien, con una casuística de 20 casos, asigna un $30 \%$ de mortalidad a la difteria maligna. Creemos que la diferencia se debe a la inclusión de difterias graves en el grupo de las malignas, ya que en la casuistica a que nos referimos no se exigió, para aceptar el diagnóstico de dífteria maligna, las condiciones enumeradas. De todas ellas, sin lugar a dudas, el elemento más importante y que imprime a este tipo de difteria su sello característico, es el cuadro de profunda toxemia que hace que el enfermo esté por momentos extraordinariamente excitado para caer luego en estado de sopor y aún de obnubilación sensorial. Para nosotros, este tipo de difteria mata inexorablemente por toxemia y es así como en nuestra casuística no contamos con ningún caso que haya sobrevivido.

"Hacemos especial hincapié en la necesidad de que exista el cuadro tóxico, ya que muchas veces, en difterias faríngeas que catalogamos como graves, se presenta uno o más de los síntomas enumerados $y, \sin$ embargo, hacen una evolución favorable en cuanto a la vida, aun cuando presenten complicaciones; lo mismo vale para aquellos casos en que la difteria por su extensión compromete la vía respiratoria en forma intensa, originando síndromes obstructivos graves $y$ a veces mortales, pero en los cuales creemos que la gravedad está condicionada por la hipo o anoxia determinada principalmente por la obstrucción.

Otro aspecto de la variedad maligna de la difteria es su duración. Según ruestra experiencia ella no fué superior a 10 días, de tal modo que, cuando una difteria grave sobrepasa este tiempo de evolución, podría desconfiarse del diagnóstico de difteria maligna.

Finalmente, hay que tener presente el pronóstico casi siempre fatal de este ti. po clínico de difteria. Sin lugar a dudas que la precocidad del tratamiento con suero puede tener importancia en otras formas de difteria, pero en la forma maligna a la que nos referimos, ni la precocidad en la aplicación de antitoxina, ni las grandes dosis de ella o su repetición, ni la asociación con antibióticos. han conseguido en nuestros casos, modificar el pronóstico.

Respecto a la interpretación patogénica de este cuadro. últimamente se ha demostrado que existen ciertas cepas de $C$. diphteriae capaces de producir hialuronidasa o factor de difusión. Aun cuando no está perfectamente establecido, se cree que estas cepas serían responsables de los casos más graves de difteria.

Experimentalmente, en cuyes, se ha visto que la inoculación de toxina difté. rica asociada a hialuronidasa produce una muerte más rápida, con hemorragias de cápsulas suprarrenales $y$ lesiones extensas en el corazón y riñón.

La hialuronidasa favorecería la penetración de la toxina diftérica en los tejidos sensibles a ella ${ }^{16}$.

Basándose en estos hechos se ha pen. sado que cuando el $\mathrm{C}$. diphteriae se asocia al Streptococcus B. hemolítico determina un cuadro más grave de difteria i 7 , debido a que el Streptococeus B. hemolítico es uno de los gérmenes que producen más alta concentración de hialuronidasa

\section{RESUMEN}

Se presenta la experiencia sobre difteria maligna, a través de 19 casos atendidos en el Servicio de Infecciosos del Hospital "Manuel Arriarán", desde 1946 hasta 1954.

Se analizan las caracteristicas generales y los datos más importantes obtenidos mediante la anamnesis, así como la sintomatología que consideramos típica de esta forma de difteria.

Se da a conocer los resultados de los exámenes de laboratorio practicados en estos enfermos, los que no fueron muy numerosos por la gravedad extrema en que se encontraban $y$ por la brevedad de su permanencia en el Servicio, hechos que 
al mismo tiempo impidieron hacer un estudio seriado o planificado.

El tratamiento de urgencia fué la principal preocupación del médico, en todas las oportunidades. Siempre se usó antitoxina diftérica en grandes dosis, se administraron antibióticos y se tomaron las medidas de enfermería de rigor.

Los hallazgos de Anatomía Patológica revelaron que fueron el corazón y los riñones los organos dañados en forma más constante.

Por último se hace un comentario sobre lo que en nuestro concepto debe llamarse difteria maligna, la que apartándola de las formas graves de difteria, dan en nuestra experiencia, una mortalidad de $100 \%$.

\section{SUMMARY}

\section{Malignant Drphteria.}

The experience based on 19 cases of malignant diphteria, hospitalized in the Department for Infectious Diseases of the "Manuel Arriaran" Hospital, from 1946 till 1954, is reported.

The general features and most important data of the history and typical symp. toms of this form of diphteria are analyzed.

The findings got from laboratory tests made in these patients are exposed. The seriousness of all these cases and their short stay in the hospital do not allow a planed study.

The treatment of emergency was the principal preocupation of the physician In all of the cases diphteria antitoxin in great doses, antibiotics and nursing care were used.
Post-mortem examination revealed thrt the heart and the kidneys were the organs most constantly damaged.

Finally a commentary is done about the concept of malignant diphteria, which must be differentiate from the gravis form, which has, in our experience, a mortality rate of $100 \%$.

\section{BIBLIOGRAFIA}

1. -WTEJEERHOLD. A.- "Difletia Mäigna". Arcb Hasu R. del Rio. 19:31, 1952.

2. - BATAX F y MAGGi R - "La estrichnterapia eu la fiiteria malizna". Fi llia Jlélico, 2n:41B, 1948.

3.-HLRRAY FISHER, A. y COBB, S. "clinical inanfestations of the severe form of diphteriat". Bal.

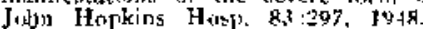

4.-PJROSPERI, P, y BORSELLI. ]. - "Eitadistica v consideraciones ctinicas de la lifteria en el pering

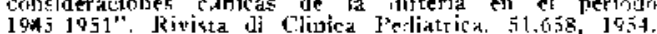

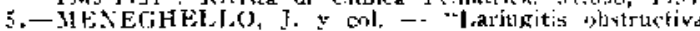

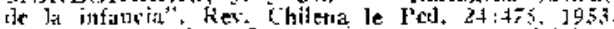

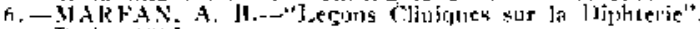
Taris, 1 ito

J,-2,Et'NUA, J, I. - "l.a difteria maligna". Anales de

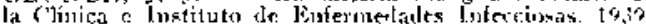

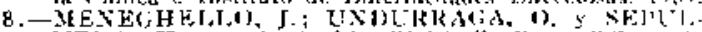

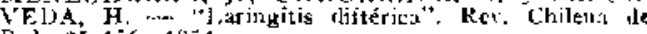
Peil. $25: 156,1954$

9.-RIE

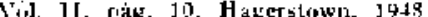

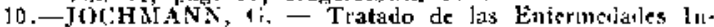
Jectiusas, pấ. 462, 19.35

11.-HF.RNIXG, A. - "On the antilacterial action or a trenicilliun with sjecial reference to dieir use in the jso'ation of B. lufhenzae". Brit. J. Expret. P'itth. :0: 226. 1929.

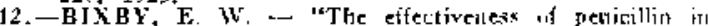
the trealment of masugharynoteal diphterid". Am. J. A1. sc. $215: 504,1948$.

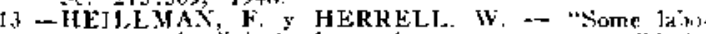
raters and clinical ohservalious on a new firtilisiti: erytiromycin". Frac. Stuff. Heet. Slave Clin, $2 \vec{f}: 385$ 19.52

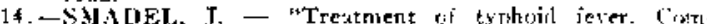
bincd therapy with cortione and thiaranthenical". Anst. lit lied. $34: 1,1953$

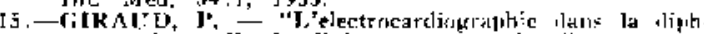
terie maligue. Etude clinique ex prounostique". Lá preske Vléticale 41:571, 1946.

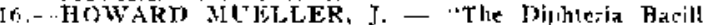
2.11 the Dirhterwirls", in R. Thalos "Bacterial 27, niycotic infection of nian". J. B. Lipmincot Co, 2nul, Ed. Philadelplia, 1952

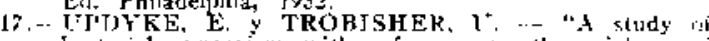
Facterial sytrescion with referetre to the eliolory ui malignant diphteriae". J. Bateriwhogy. 54:620, 197\%. 